\title{
General Relativistic Constraints on Emission Models of Anomalous X\#Ray Pulsars
}

\section{Citation}

DeDeo, Simon, Dimitrios Psaltis, and Ramesh Narayan. 2001. “General Relativistic Constraints on Emission Models of Anomalous X\#Ray Pulsars." The Astrophysical Journal 559 (1): 346-52. https://doi.org/10.1086/322283.

\section{Permanent link}

http://nrs.harvard.edu/urn-3:HUL.InstRepos:41384935

\section{Terms of Use}

This article was downloaded from Harvard University's DASH repository, and is made available under the terms and conditions applicable to Other Posted Material, as set forth at http:// nrs.harvard.edu/urn-3:HUL.InstRepos:dash.current.terms-of-use\#LAA

\section{Share Your Story}

The Harvard community has made this article openly available.

Please share how this access benefits you. Submit a story.

Accessibility 


\author{
GENERAL RELATIVISTIC CONSTRAINTS ON EMISSION MODELS OF \\ ANOMALOUS X-RAY PULSARS \\ Simon DeDeo, Dimitrios Psaltis, and Ramesh Narayan \\ Harvard-Smithsonian Center for Astrophysics, 60 Garden St., Cambridge, MA 02138; sdedeo, dpsaltis, \\ narayan@cfa.harvard.edu \\ Draft version November 17, 2018
}

\begin{abstract}
Most models of anomalous X-ray pulsars (AXPs) account for the observed X-ray spectra and pulsations by means of radiation processes that occur on the surfaces of neutron stars. For any such model, general relativistic deflection of light severely suppresses the amplitude of the observed pulsations. We calculate the expected pulsation amplitudes of AXPs according to various models and compare the results with observations. We show that the high $(\$ 70 \%)$ pulse amplitudes observed in some AXPs can be accounted for only if the surface emission is localized (spot radius $\left.<40^{\circ}\right)$ and strongly beamed $\left(\sim \cos ^{n} \theta^{\prime}\right.$ with $n \gtrsim 2$, where $\theta^{\prime}$ is the angle to the normal). These constraints are incompatible with those cooling and magnetar models in which the observed X-rays originate as thermal emission from the neutron-star surface. Accretion models, on the other hand, are compatible with observations for a wide range of parameters. Finally, definitive conclusions cannot be reached on magnetospheric models, since their localization and beaming properties are not well understood.
\end{abstract}

Subject headings: accretion, accretion disks — relativity — stars: neutron — X-rays: stars

Submitted to The Astrophysical Journal.

\section{INTRODUCTION}

Among pulsating compact X-ray sources are a small subset with pulsation periods between 6 and 12 seconds, soft spectra, and no identifiable companions. The first suggestion that these objects might form a separate class of neutron stars - later called the anomalous X-ray pulsars (AXPs) - was made by Mereghetti \& Stella (1995), who proposed that they might be powered by accretion from a very low mass companion (this was also earlier hinted at by Hellier 1994). The lack of optical counterparts, however, and the absence of observable Doppler shifts in the frequency of the X-ray pulses led van Paradijs, Taam, \& van den Heuvel (1995) to favor a different accretion model for AXPs. According to their suggestion, material from a fossil accretion disk, possibly the debris of a disrupted binary companion after a period of common-envelope evolution, is being accreted by a solitary neutron star. Recently, a similar model has been proposed by Chatterjee et al. (2000), in which the accreting material is supplied by the post-supernova fallback material from the neutron star progenitor itself.

In a different class of models, AXPs are considered to be isolated neutron stars, spinning down by magnetic dipole radiation. Because of their unusually high period derivatives, a simple application of the relationship between the dipole magnetic field strength and the spin-down rate implies a field strength for these objects of $\sim 10^{14}-10^{15} \mathrm{G}$ (see, e.g., Thompson \& Duncan 1996). Two main types of models that rely upon the presumed high magnetic field of the stars have been proposed. Thompson \& Duncan (1996; see also Duncan \& Thompson 1992) suggested that the released energy may be drawn from the decay of the magnetic field itself and from differential movements in the stellar crust. This model also serves to explain the bursts observed from soft gamma-ray repeaters as being produced by larger-scale magnetospheric phenomena. Because of such models, AXPs are often called "magnetars" and are grouped into the same class of sources as the soft gammaray repeaters (see, e.g, Thompson \& Duncan 1995, 1996; also Hurley 2000 for a review of SGR properties and models). In the alternative high magnetic-field model, Heyl \& Hernquist (1997a, 1997b) suggested that AXPs draw their energy from the residual thermal energy of the star itself.

All these models face a number of difficulties. For example, if AXPs are powered by accretion from a stellar companion, the absence of detectable Doppler shifts in the arrival of X-ray pulses cannot be easily explained(Mereghetti et al. 1998). If accretion is from either a companion or a fossil disk, the optical fluxes one would expect directly from the disk or due to X-ray reprocessing are too high compared to the observed upper limits (see, e.g., Perna, Hernquist, \& Narayan 2000; Hulleman et al. 2000). On the other hand, in the magnetar model the absence of bright faster AXPs and the observed variations in spindown rates are hard to account for (see, e.g., Baykal \& Swank 1996; Marsden et al. 2000; see, however, Heyl \& Hernquist 1999; Melatos 1999).

In all the above models of AXP, nearly all of the X-ray emission is produced at the surface of the neutron star. It is well known that strong gravitational fields tend to smooth out the variability produced by a spinning compact star, even if the emission is highly localized in bright spots (Pechenick, Ftaclas, \& Cohen 1983). Indeed, the Xray pulse amplitudes of the three radio pulsars that show thermal emission from their surfaces are only $\lesssim 30 \%$ (see, e.g., the discussion in Page 1995; Harding \& Muslimov 1998). This is in contrast to the non-thermal emission from radio pulsars (which is magnetospheric in origin) and from accretion-powered X-ray pulsars (which is from collimated accretion columns) which often show pulse ampli- 
tudes as high as $\sim 90 \%$ (see, e.g., Nagase 1989). In this respect, AXPs are similar to accretion-powered pulsars, showing X-ray pulse amplitudes anywhere between $\sim 10 \%$ and $\sim 70 \%$.

We study in this paper a set of variability diagnostics that may be used in constraining emission models of AXPs. We examine three parameters: the pulse fraction observed at infinity, which is a measure of the overall amplitude of the variations, and the Fourier amplitudes at the first and second harmonics of the neutron-star spin frequency. We find tight constraints on the properties of magnetar models. Most importantly, we are unable to reproduce the observed variability properties of AXPs with thermal cooling models.

\section{FORMALISM}

In order to determine how relativistic effects suppress or enhance variability amplitudes, we need to consider curved photon paths from the surface of the star to an observer at infinity. Since the objects under consideration are rotating slowly, we use the Schwarzschild spacetime, which is appropriate for a non-spinning mass, and ignore effects such as relativistic frame dragging, which are important only for rapidly spinning objects. In this section, we outline the basic ingredients of our method, drawing on the work of Pechenick et al. (1983).

For each model we specify both the brightness distribution over the surface of the star and the effective beaming of radiation, i.e., we specify the specific intensity integrated over photon energy, $I\left(\theta, \phi, \theta^{\prime}\right)$, that emerges from each point on the stellar surface with polar coordinates $(\theta, \phi)$ at an angle $\theta^{\prime}$ with respect to the normal.

In the current analysis we consider a number of different, physically motivated, mathematical expressions for the dependence of the emerging specific intensity on $\theta^{\prime}$ (hereafter called the beaming function). This allows us to explore a large parameter space and draw conclusions that do not depend strongly on any particular emission model. We consider isotropic emission, i.e., no dependence on $\theta^{\prime}$, as well as the beaming described by the Hopf function (Chandrasekhar 1950, eq. [III.50])

$$
I\left(\theta, \phi, \theta^{\prime}\right)=I_{0}(\theta, \phi)\left(\sum_{a=1}^{3} \frac{L_{a}}{1+k_{a} \cos \theta^{\prime}}-\cos \theta^{\prime}+Q\right),
$$

where the parameters $L_{a}, k_{a}$, and $Q$ are given in Chandrasekhar (1950; Table III.VII). The Hopf function describes the beaming of radiation emerging from a scattering atmosphere heated from below and is, therefore, suitable for a weakly-magnetic $\mathrm{H}-\mathrm{He}$ atmosphere at energies $\gtrsim 1 \mathrm{keV}$ (Zavlin et al. 1998).

For photon energies near the cyclotron energy $\left(E_{\text {cyc }} \simeq\right.$ $11.6\left[B / 10^{12} \mathrm{G}\right] \mathrm{keV}$ ), the beaming function for a magnetic atmosphere does not always decrease monotonically away from the radial direction. Indeed the beaming function may have a local minimum at small angles from the normal (Zavlin et al. 1995). However, for a dipole magnetic field this effect is not very significant and results in a rather small pulse fraction $(\lesssim 30 \%)$ even when general relativistic effects are not taken into account (see Zavlin et al. 1995). Moreover, the cooling models of AXPs discussed here

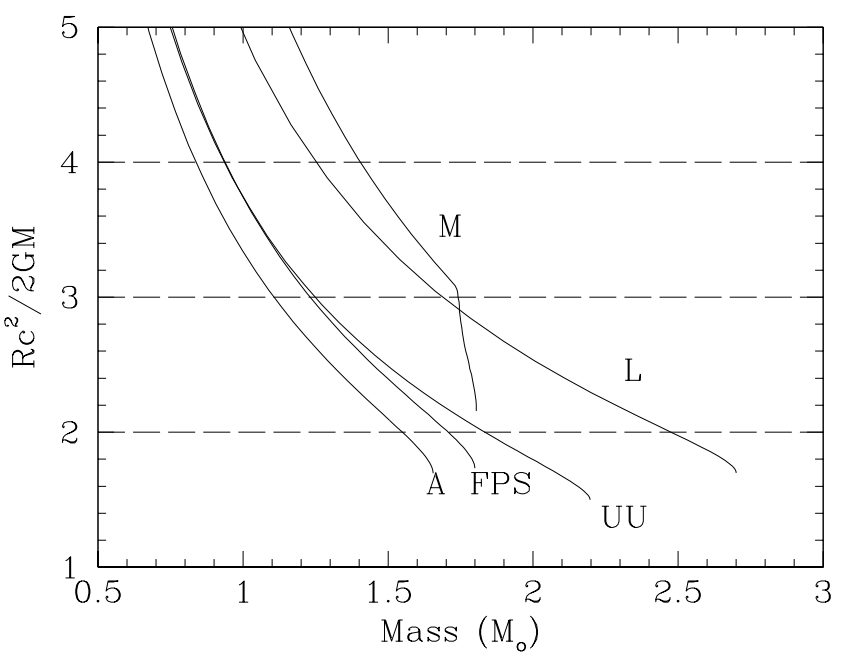

FIG. 1.- Neutron star radii, in units of the Schwarzchild radius, for different neutron star masses and equations of state (A: Pandharipande 1971; FPS: Lorenz, Ravenhall, \& Pethick 1983; UU: Wiringa, Fiks, \& Frabrocini 1988; L: Pandharipande \& Smith 1975b; M: Pandharipande \& Smith 1975a).

require that the magnetic field of the neutron star is $\sim 10^{14}-10^{15} \mathrm{G}$ and hence that the cyclotron energy is $\sim 1-10 \mathrm{MeV}$, i.e, much larger than the $\sim 1 \mathrm{keV}$ photon energies that are of interest here. For such energies, it is reasonable to consider beaming functions that decrease monotonically away from the normal to the surface (Zavlin et al. 1995).

In models of accretion columns, the interaction of radiation with a plasma in the strong magnetic field of the neutron star, as well as the possibility of radiation being obscured by the accretion column (see, e.g., Riffert et al. 1993) leads to a much sharper beaming. For this reason, we also consider beaming functions of the form

$$
I\left(\theta, \phi, \theta^{\prime}\right)=I_{0}(\theta, \phi) \cos ^{n} \theta^{\prime} .
$$

According to Nagel (1981; see also Meszaros \& Nagel 1985), the radiation pattern emerging from an accretion column at low accretion rates is described quite well with $n \simeq 2-3$.

Given a model for the X-ray emission from the stellar surface, the flux measured by an observer at distance $d$, whose polar coordinates with respect to the stellar rotation axis are $(\beta, \Phi)$, is given by (cf. Pechenick et al. 1983)

$$
\begin{aligned}
F_{\infty}(\beta, \Phi)= & \left(\frac{R}{d}\right)^{2}\left(\frac{M}{R}\right)^{2}\left(1-\frac{2 M}{R}\right)^{2} \\
& \times \int_{x=0}^{x_{\max }} \int_{y=0}^{2 \pi} I\left(\theta, \phi, \theta^{\prime}\right) x d x d y .
\end{aligned}
$$

In the above equation $\theta, \phi$, and $\theta^{\prime}$ depend implicitly on the angles $x$ and $y$ as described in Pechenick et al. (1983), $x_{\max } \equiv(R / M)(1-2 M / R)^{-1 / 2}, M$ and $R$ are the neutron star mass and radius, and we have set $c=G=1$. The double integral has an integrable pole at $x_{\max }$. In the calculations presented here we have evaluated this integral to an accuracy of $10^{-3}$ using Romberg integration of the fifth order. 
AXP PROPERTIES

\begin{tabular}{clcc}
\hline Label & Source Name & Pulse Fraction & $I_{2} / I_{1}$ \\
\hline A & 1E 1048.1-5937 & 0.76 & 0.15 \\
B & 1E 1841-045 & 0.15 & 0.54 \\
C & AX J1845.0-0258 & 0.63 & 0.14 \\
D & 1RXS J170849.0-400910 & 0.50 & 0.40 \\
E & 4U 0142+61 & 0.17 & 0.70 \\
F & 1E 2259+586 & 0.35 & 1.35 \\
\hline
\end{tabular}

References.- A: Oosterbroek et al. $1998(S A X)$; B: Gotthelf et al. $1999(A S C A+S A X)$; C: Torri et al. 1998 (ASCA); D: Sugizaki et al. 1997 ( $A S C A)$; E: Israel et al. 1999 (SAX); F: Iwasawa et al. 1992 (GINGA).

The degree of suppression of the pulsation amplitude depends sensitively on the compactness of the neutron star. Figure 1 shows the ratio $p \equiv R c^{2} / 2 G M$ for different neutron-star masses and equations of state. Based on this figure, we limit our parameter study to $p=2,3$, and 4 ; larger values of $p$ would correspond to unrealistically light neutron stars, even for the stiffest proposed equations of state (i.e., $\lesssim 1.4 M_{\odot}$ even for equation of state $\mathrm{M}$ ).

\section{PULSATION AMPLITUDES}

The pulsation amplitudes observed in anomalous X-ray pulsars allow us to place constraints on models of X-ray emission from their surface. In this paper, we do not attempt to fit particular observed pulse profiles but rather try to set general constraints on large classes of models. For this reason, we only consider the pulse fraction, defined as (cf. eq.|3])

$$
P F \equiv \frac{F_{\infty}^{\max }-F_{\infty}^{\min }}{F_{\infty}^{\max }+F_{\infty}^{\min }},
$$

and the Fourier amplitudes of the harmonics of the pulse frequency, defined by

$$
F_{\infty}(\beta, \Phi)=I_{0}(\beta)+I_{1}(\beta) \cos (\Phi)+I_{2}(\beta) \cos (2 \Phi)+\ldots
$$

Table I shows the observed pulse fractions and harmonic content for six AXPs, as inferred approximately from published lightcurves. We see that half of the known systems have pulse fractions of 0.5 or larger, with the largest being $\sim 0.7$. As we discuss below, this fact provides strong constraints on some models of AXPs.

\subsection{Cooling of Magnetic Neutron Stars}

The brightness distribution on the surface of a stronglymagnetic cooling neutron star depends on the local magnetic field strength $B$ and its angle $\psi$ with respect to the local radial direction (see, e.g., Heyl \& Hernquist 1998). The flux emerging from a spot on the stellar surface is $\sim B^{m} \cos ^{2} \psi$, with $m \simeq 0.4$ approximating well the numerical results (Heyl \& Hernquist 1998). For a dipole stellar field we, therefore, use

$$
I_{0}(\theta, \phi) \sim I_{0} \frac{\cos ^{2} \theta}{\left(3 \cos ^{2} \theta+1\right)^{1-m / 2}}, \quad m=0.4,
$$

where $(\theta, \phi)$ are polar coordinates on the stellar surface with respect to the magnetic axis.
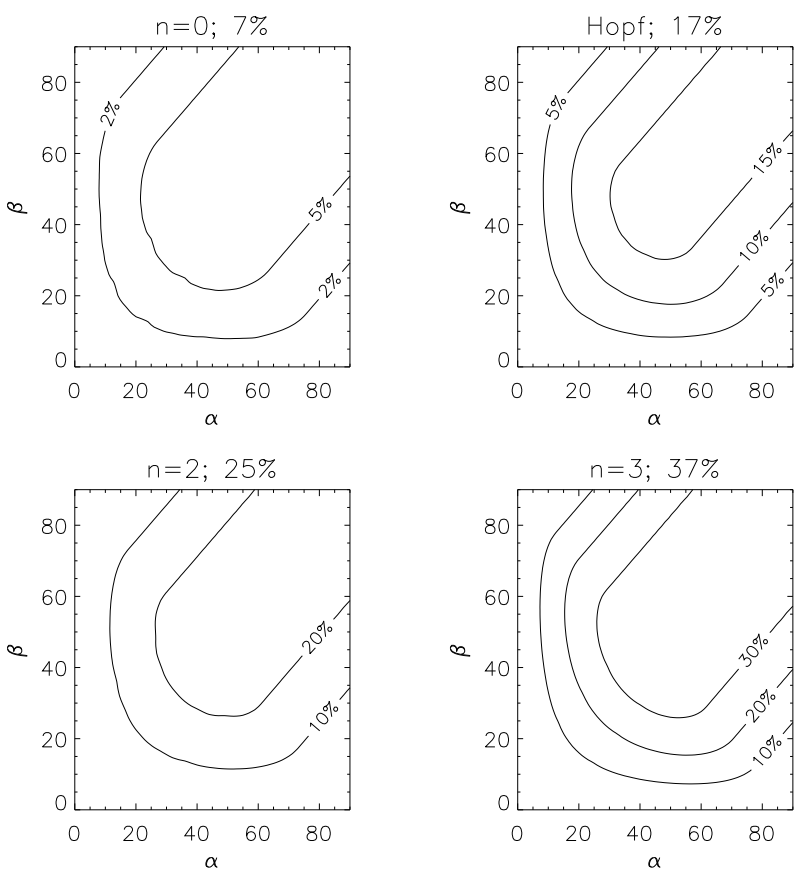

FIG. 2.- Pulse fractions calculated for cooling neutron stars with a dipole magnetic field, and different magnetic inclinations $(\alpha)$ and orientations of the observer $(\beta)$ with respect to the rotation axis. The different panels correspond to an isotropic beaming function $(n=0)$, a Hopf function, and beaming functions that are increasingly more peaked towards the radial direction $(n=2,3)$.

Figure 2 shows the predicted pulse fractions for a cooling neutron-star model, for different choices of the angle $\alpha$ between the magnetic dipole axis and the rotation axis and the angle $\beta$ between the light of sight to the observer and the rotation axis, as well as for different beaming functions. Some general features of this figure are worth noting. For example, the predicted pulse fraction remains unchanged when the magnetic inclination and the inclination of the observer are interchanged. This is true because the flux at infinity measured at any give pulse phase $\Phi$ depends only on the angular distance

$$
\theta_{0}=\cos ^{-1}(\sin \alpha \sin \beta \cos \Phi+\cos \alpha \cos \beta)
$$

between the magnetic axis and the direction to the observer, which is symmetric in $\alpha$ and $\beta$. Furthermore, the predicted pulse fraction is maximum when $\alpha=\beta$. The overall maximum occurs when $\alpha=\beta=90^{\circ}$ and the pulse fraction is zero when either $\alpha=0^{\circ}$ or $\beta=0^{\circ}$.

The most important result from Figure 2 is that even for the most favorable geometry (i.e., $\alpha=\beta=90^{\circ}$ ) and for strong beaming (i.e., $n=3$ ), the pulse fraction does not exceed $37 \%$. The reason is that the surface brightness distribution (6) is too smooth. Assuming a less relativistic neutron $\operatorname{star}(R / 2 M=4)$, or even a significantly stronger dependence of the emerging flux on the local magnetic field $(m=1$ in eq. [6]) results in only a modest increase of the maximum pulse fraction $(51 \%$ and $50 \%$, respectively, for $n=3$ ). We thus conclude that neutron star cooling models with the surface emission described by equation (6) cannot reproduce the large pulse fractions observed from AXPs. 


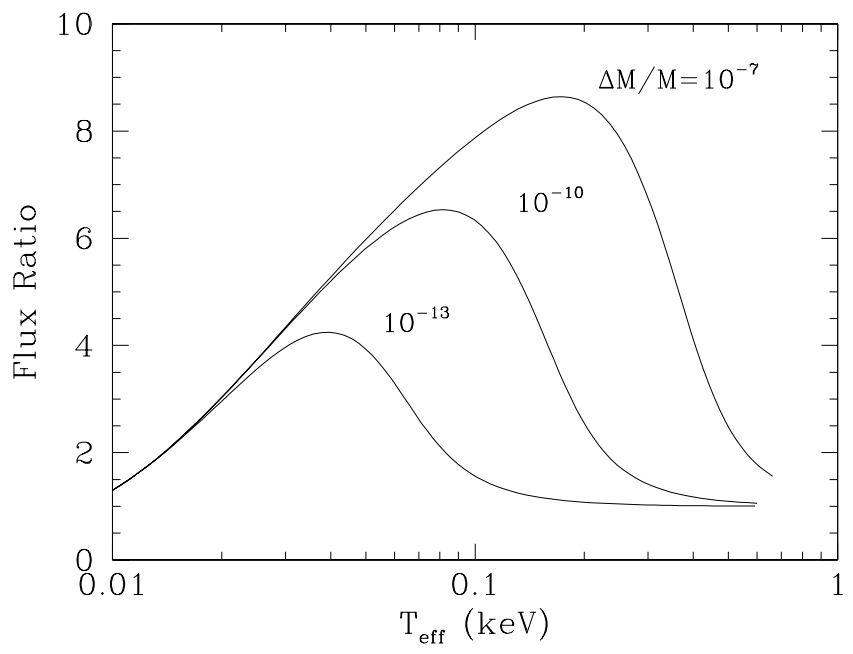

FIG. 3.- Ratio of the bolometric flux emerging from an atmosphere with a given mass fraction $(\Delta M / M)(\Omega / 4 \pi)$ (in terms of the neutron-star mass $M$ ) of $\mathrm{H}-\mathrm{He}$ matter accreted over a fraction $\Omega / 4 \pi$ of the stellar surface, to the bolometric flux from a purely iron atmosphere with the same core temperature (after Chabrier et al. 1997).

\subsection{Localized Thermal Emission}

Thermal emission from the neutron-star surface can, in principle, be more localized than indicated in equation (6), if it is confined mostly around the stellar magnetic poles. For example, metallicity gradients on the surface of a cooling neutron star, possibly produced by magneticallychannelled fallback material during the supernova explosion, can lead to larger effective temperatures near the magnetic poles than in the magnetic equator (see, e.g., Pavlov et al. 2000). Alternatively, non-uniform heating of the neutron-star atmosphere, e.g., caused by magnetic field decay or crustal heating (see, e.g., Thompson \& Duncan 1996), can lead to more localized thermal emission from its surface.

We estimate the magnitude of the first effect using the analytic expressions for neutron-star atmosphere models given by Chabrier, Potekhin, \& Yakovlev (1997). We assume that a fraction $(\Delta M / M)(\Omega / 4 \pi)$ of light element material has accumulated only over a fraction $(\Omega / 4 \pi)$ of the stellar surface. We neglect the fact that the surface layers of the neutron star are in the liquid phase and hence lateral diffusion may smooth out the metallicity gradient (L. Hernquist, private communication). For a given core temperature we then calculate the ratio $f$ of the bolometric flux emerging from the light-element region of the surface to the bolometric flux from the region consisting purely of iron. This flux contrast is shown in Figure 3 , for different values of the mass fraction of the accreted atmosphere. The flux contrast does not further increase, for $(\Delta M / M)(\Omega / 4 \pi) \gtrsim 10^{-7}$, because the base of the accreted layer reaches densities that are high enough $\left(\sim 10^{10} \mathrm{~g} \mathrm{~cm}^{-3}\right)$ for it to be part of the isothermal core. Although the above models are strictly valid only for weakly magnetic neutron stars, the flux ratios are significantly more sensitive to the composition of the neutron-star envelope than the magnetic field strength (cf. Heyl \& Hernquist 1997b).

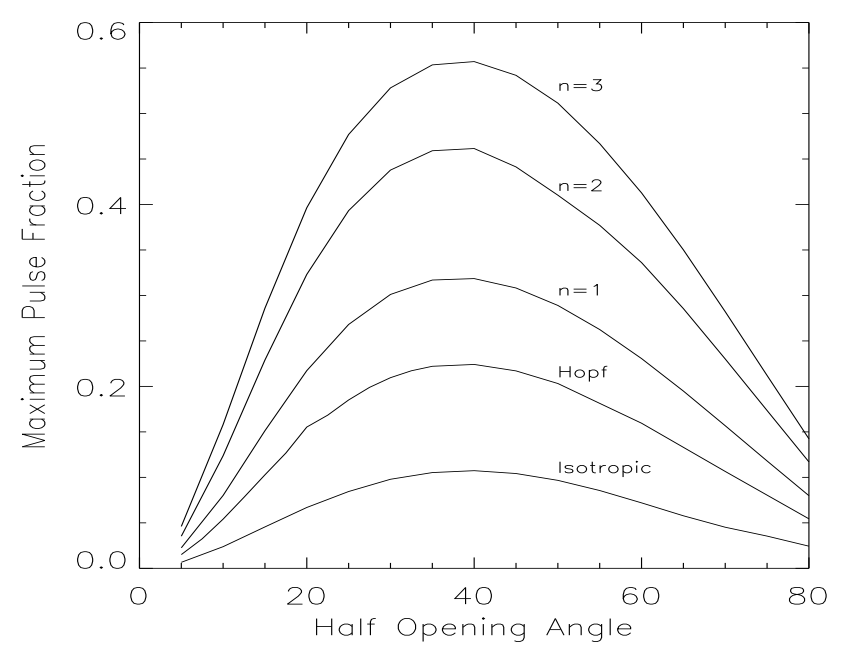

Fig. 4.- Calculated pulse fractions for a neutron star with $R / 2 M=3$, as a function of the half-opening angle of each polar cap, for different beaming functions, and a flux contrast of 9 between the polar caps and the rest of the stellar surface.

According to Figure 3, the expected flux contrast between the polar caps and the rest of the stellar surface can be at most $f \sim 9$ and the contrast depends very weakly on the amount of accreted material. For the flux contrast to attain its maximum value, the polar caps must accrete $\Delta M \gtrsim 10^{-7} M_{\odot}$, while the rest of the surface must accumulate more than three orders of magnitude less material. Even if these conditions were met and even if the photons emerged from the stellar surface strictly radially, the pulse fraction would be at most $(f-1) /(f+1) \sim 0.8$.

Any realistic beaming function reduces this upper bound significantly below the highest observed pulse fraction for AXPs $(\sim 0.7$; see Table I), even for mildly relativistic neutron stars. This is demonstrated in Figure 4, which shows the maximum pulse fraction of radiation emerging from a neutron star with $R / M=3$, as a function of the angular radius of each polar cap, for various realistic beaming functions, and an emerging flux from the polar caps that is nine times larger than the rest of the stellar surface. For small polar-cap sizes, the radiation flux emerging from the caps is only a small perturbation to the total brightness of the star and hence the pulse fraction is small. With increasing cap size the relative contribution of the caps to the brightness of the star increases, leading to an increase of the pulse fraction, until the caps cover a large enough fraction of the stellar surface and the pulse fraction drops again. For the flux contrast and neutron star radius used here, the maximum pulse fraction occurs when the polar-cap size is $\sim 40^{\circ}$ and this maximum value is $\sim 55 \%$. Clearly, no realistic model of this kind can fit the pulse fraction of $\sim 0.7$ seen in 1E 1048.1-5937.

The pulse fraction can be further enhanced if the fluxes emerging from two antipodal polar caps are unequal, e.g., because of uneven fallback or crustal heating. As the contrast between the two polar caps increases, the pulse fraction becomes larger, but only at the expense of the Fourier amplitudes at the harmonics of even order. This is shown in Figure 5 , where the predicted ratio $I_{2} / I_{1}$ is plotted against the pulse fraction for $R / M=3$, two 


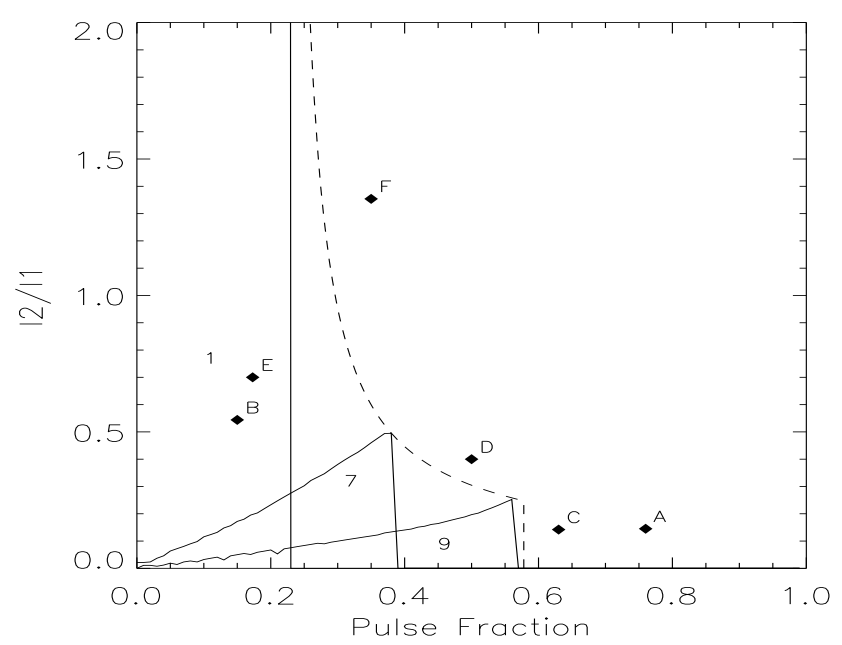

FIG. 5.- Ratio $I_{2} / I_{1}$ of the Fourier amplitudes at the second harmonic and fundamental of the pulsar spin frequency (cf. eq. 150) as a function of the total pulse fraction (cf. eq. 岾). The calculations correspond to $R / M=3$, a configuration of two antipodal polar caps with a half-opening angle of 40 degrees but different emerging fluxes, and beaming described by the Hopf function. The solid lines outline the area in the parameter space allowed for specific ratios of fluxes emerging from the two polar caps. The dashed line represents the maximum ratio $I_{2} / I_{1}$ that corresponds to a given pulse fraction; for such a configuration, no system is allowed to lie to the right of the dashed line. The diamonds correspond to the observed properties of AXPs (cf. Table 1).

antipodal caps with a half-opening angle of 40 degrees, and beaming described by the Hopf function. For these calculations, the two caps are assumed to have different emerging radiation fluxes, with the flux of the brightest set to 9 times the flux emerging from the rest of the neutron-star surface. For any given pulse fraction, there exists a maximum value that the ratio $I_{2} / I_{1}$ can attain, shown by the dashed line in Figure 5. As a result, detection of a source with a large pulse fraction and significant amplitude at the even order harmonics can exclude such a configuration.

The observed properties of some AXPs are not consistent even with a model with such unequal polar caps (Figure 5). For example, the source $1 \mathrm{E} 2259+586$ is characterized by a large pulse fraction and high harmonic content that cannot be achieved by any of the configurations considered here. It does not, therefore, appear plausible for thermal emission from the stellar surface to account for the variability properties of AXPs. Achieving the kind of variability observed is possible only when the emission is both localized and strongly beamed.

\subsection{Accretion Onto Magnetic Neutron Stars}

In accretion models of AXPs (see, e.g., Mereghetti \& Stella 1995; van Paradijs et al. 1996; Chatterjee et al. 1999), a large fraction of the X-ray emission may be produced mainly at localized "hot spots" where the accretion columns meet the stellar surface. In order to take such configurations into account, we describe the surface brightness distribution with two circular antipodal spots, with a brightness that is constant over their surface area. We denote by $\alpha$ the angular distance of the center of each spot from its closest rotation pole.
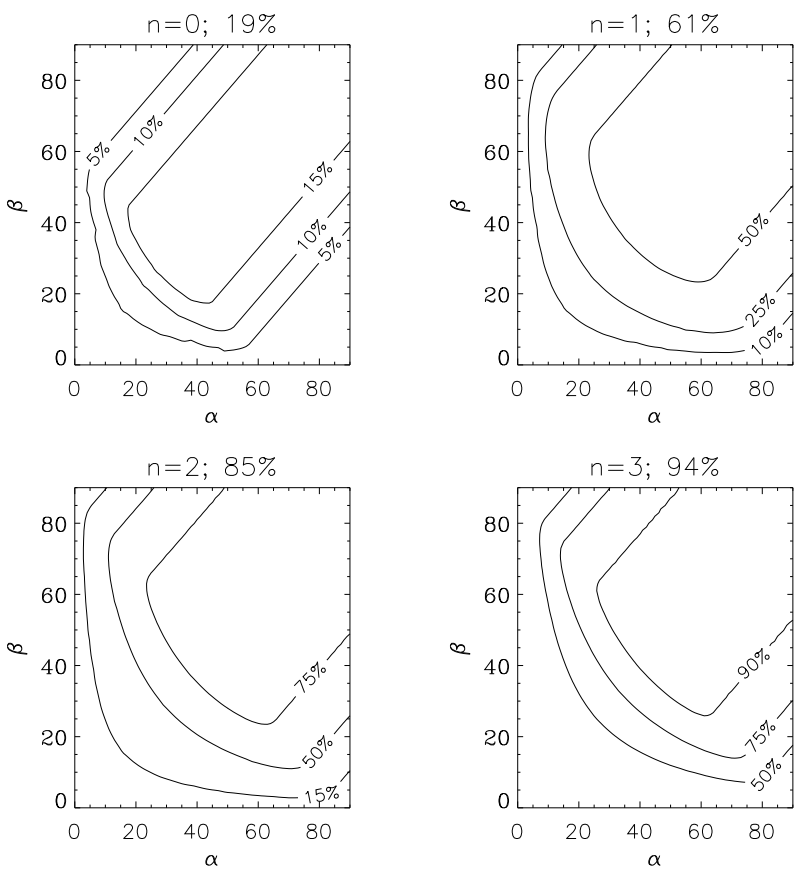

FIG. 6.- Calculated pulse fractions for a neutron star with $R / 2 M=3$, a hot-spot half-opening angle of 5 degrees and a $\cos ^{n} \theta^{\prime}$ beaming function, with $n=0-3$. The maximum value of the pulse fraction is written above each panel.
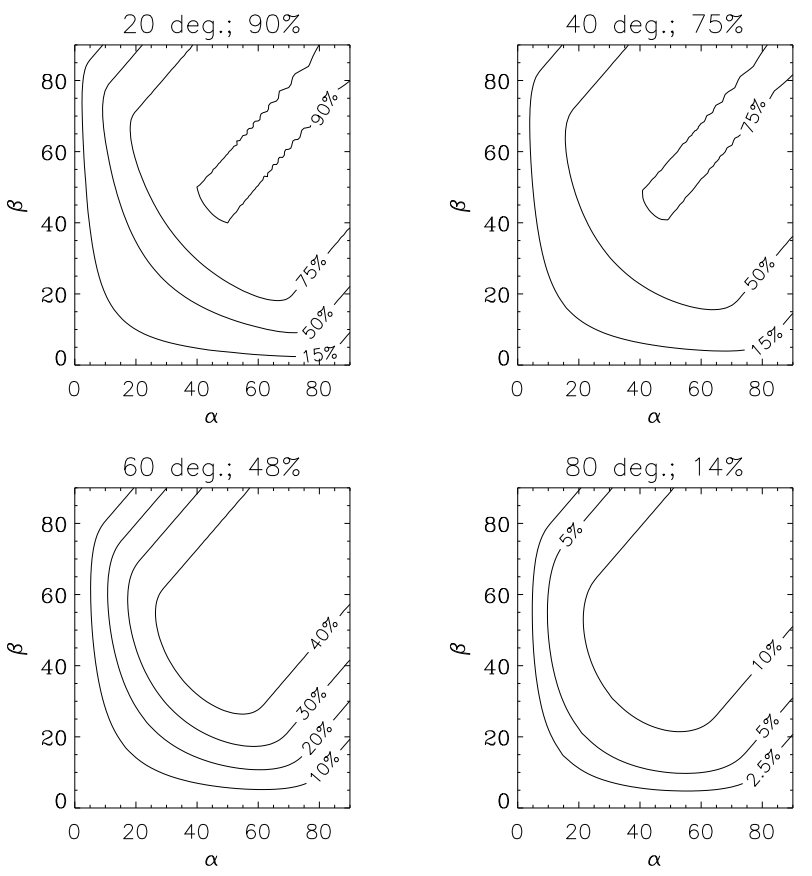

FIG. 7.- Calculated pulse fractions for a neutron star with $R / 2 M=3$, a $\cos ^{3} \theta^{\prime}$ beaming function, and different hot-spot halfopening angles. The maximum value of the pulse fraction is written above each panel.

If AXPs are powered by accretion from a geometrically thin disk, the half-opening angle of each polar cap can be very small. We estimate the size of each polar cap as the angular distance on the stellar surface from the magnetic axis of the footpoint of the last magnetic field line that 

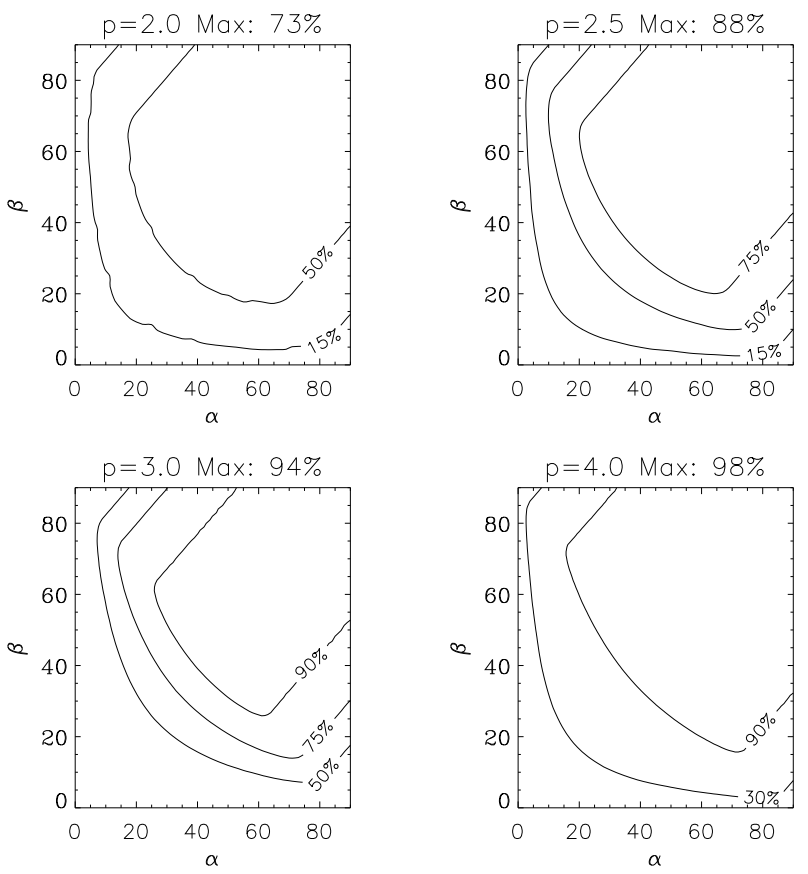

FIG. 8.- Calculated pulse fractions for different neutron-star radii $(p=R / 2 M)$, a hot-spot half-opening angle of 5 degrees, and a $\cos ^{3} \theta^{\prime}$ beaming function. The maximum value of the pulse fraction is written above each panel.

penetrates the accretion disk. Given that all AXPs are observed to be spinning down, their accretion disks must be truncated near the outer corotation radius (see, e.g., Ghosh \& Lamb 1979)

$$
\begin{aligned}
R_{\mathrm{co}} & \equiv\left(\frac{G M P^{2}}{4 \pi^{2}}\right)^{1 / 3} \\
& \simeq 550 R\left(\frac{R}{10^{6} \mathrm{~cm}}\right)^{-1}\left(\frac{M}{1.4 M_{\odot}}\right)^{1 / 3}\left(\frac{P}{6 \mathrm{~s}}\right)^{2 / 3}
\end{aligned}
$$

where $P$ is the spin period of the pulsar. For a dipole magnetic field, the quantity $\sin ^{2} \theta / r$, with $\theta$ measured from the magnetic pole, remains constant along a field line, and therefore the half-opening angle of the polar cap is $\sim\left(R / R_{\mathrm{co}}\right)^{1 / 2} \simeq 2.5^{\circ}$.

Figure 6 shows the pulse fraction calculated for a model with two identical hot spots, a half-opening angle of 5 degrees, and various beaming functions (see eq. (2|). Because of the general relativistic deflection of light, we see that even such a small spot cannot produce a $\sim 70 \%$ pulse fraction, unless there is significant beaming $(n \gtrsim 2)$. On the other hand, if the emerging radiation is strongly beamed towards the radial direction, a large pulse fraction can be achieved for a wide range of polar cap sizes $\left(\lesssim 40^{\circ}\right.$; Fig. 7 ) and for all realistic neutron-star radii (Fig. 8).

\section{CONCLUSIONS}

In this paper we have used the high pulse amplitudes observed from a number of AXPs to constrain the properties of their emission mechanism. We find that the observations can be accounted for only if the surface emission is localized (half-opening angle $<40^{\circ}$ ) and strongly beamed

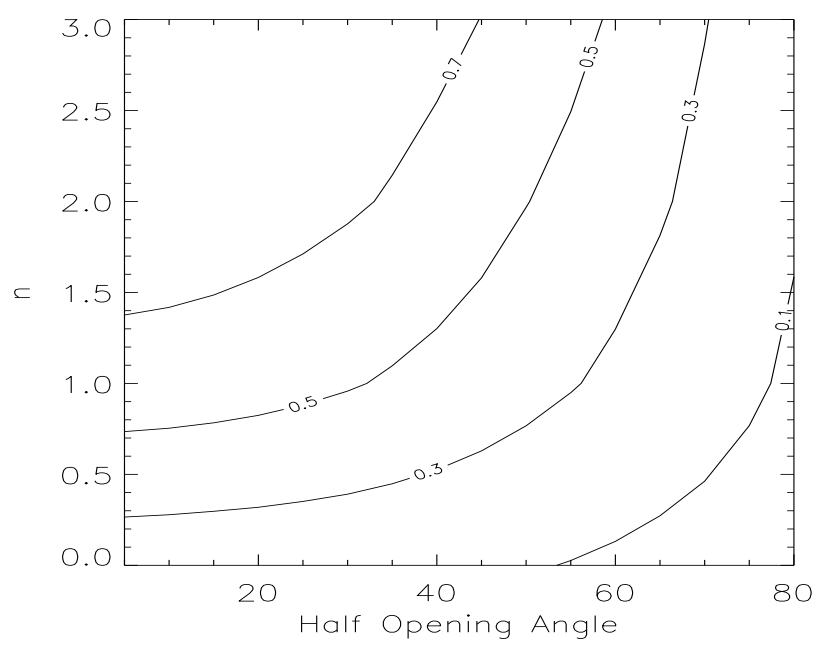

FIG. 9.- Contours of maximum pulse fraction for different beaming functions $\left(\cos ^{n} \theta^{\prime}\right.$; cf. eq. 21) and half-opening angles of each hot spot. The $\simeq 70 \%$ pulse fraction observed from the source $1 \mathrm{E} 1048.1-$ 5937 restricts any viable model of AXPs to the left of the uppermost contour.

( $n \gtrsim 2$ in eq. [2]), as summarized quantitatively in Figure 9 . These constraints are a consequence of the compactness of the neutron stars and the resulting strong general relativistic deflection of photon trajectories. Our conclusions are valid for all realistic neutron star masses and radii.

The properties of individual sources offer a number of additional clues. For example, the double-peaked pulse profile of $1 \mathrm{E} 2259+586$ requires that the emission is localized around two antipodal spots on the neutron-star surface, probably associated with the magnetic poles. Furthermore, the change in the relative strength of the two peaks observed with GINGA (Iwasawa et al. 1992) implies that the pulse shape cannot be solely due to geometric effects but should also reflect a flux contrast between the two antipodal spots. Furthermore, this flux contrast should be variable and, therefore, cannot be caused by a non-dipolar magnetic field configuration or a non-uniform fallback of low metallicity material.

Such arguments, together with the constraints presented in Figures 2, 4, 5, and 9 appear to rule out thermal cooling models for AXPs. They are also inconsistent with those magnetar models in which most of the X-ray flux originates from heating in the deep surface layers of the neutron star. On the other hand, the localized emission and beaming predicted by accretion models seem to be consistent with the observations. A magnetospheric model in which the neutron-star surface is heated over localized spots by particle bombardment may also be viable, though the beaming properties of such a model are unknown.

We thank Deepto Chakrabarty, Lars Hernquist, Vicky Kaspi, Jessica Lackey, and Feryal Özel for many useful discussions. This work was supported in part by NSF grant AST 9820686. D. P. acknowledges the support of a postdoctoral fellowship of the Smithsonian Institution. 


\section{REFERENCES}

Baykal, A., \& Swank, J. E. 1996, ApJ, 460, 470

Chabrier, G., Potekhin, A. Y., \& Yakovlev D. G. 1997, ApJ, 477, L99

Chakrabarty, D., Pivovaroff, M.J., Hernquist L E Heyl, J.S., \& Narayan, R. 2000, ApJ, submitted astro-ph/0001026)

Chandrasekhar, S. 1950, Radiative transfer (Oxford, Clarendon Press)

Chatarjee,P Narayan, R., \& Hernquist, L.E. 2000, ApJ, in press (astro-ph/9912137)

Duncan, K.C., \& Thompson, C. 1992, ApJ, 392, L9

Ghosh, P., \& Lamb, F. K. 1979, ApJ, 232, 259

Gotthelf, E. V., Vasisht, G., \& Dotani, T. 1999, ApJ, 522, L49

Harding, A. K., \& Muslimov, A. G. 1998, ApJ, 500, 862

Hellier, C. 1994, MNRAS, 271, L21

Heyl, J \& Hernquist L F 1997a 489 L67 1997b, ApJ, 491, L95 1998, MNRAS, 300, 599
1999, MNRAS, 304, L37

Hulleman, F., van Kerkwijk, M.H., W. W. \& Kulkarni, S. R. 2000, ApJ, in press (astro-ph/000247

Hurley, K. 2000, Astr. Let. Comm., in press (astro-ph/9912061

Israel, G. L., et al. 1999, A\&A, 346, 929

Iwasawa, K., Koyama, K., \& Halpern, J. P. 1992, PASJ, 44, 9

Kaspi, V. M., Chakrabarty, D., \& Steinberger, J. 1999, ApJ, 525, L33

Lorenz, C.P., Ravenhall, D. G., \& Pethick, C. J. 1993, Phys. Rev. Lett., 70, 379

Marsden, D., Rothschild, R.E., \& Lingenfelter, R.E. 2000, ApJ, in press

Melatos, A. 1999, ApJ, 519, L77

Mereghetti, S. Stella, L. 1995, ApJ, 442, L17
Mereghetti, S., Israel, G. L., \& Stella, L. 1998, MNRAS, 296, 689

Meszaros, P., \& Nagel, W. 1985, ApJ, 299, 138

Nagase, F. 1989, PASJ, 41, 1

Nagel, W. 1981, ApJ, 251, 278

Oosterbroek, T., Parmar, A. N., Mereghetti, S., \& Israel, G. L. 1998, A\&A, 334, 925

Page, D. 1995, ApJ, 442, 273

Pandharipande, V.R. 1971, Nucl. Phys., A174, 641

Pandharipande, V. R., \& Smith, R. A. 1975a, Nucl. Phys., A237, 507 1975b, Phys. Letters, 59B, 15

Pavlov, G. G., Zavlin, V. E., Aschenbach, B., Trümper, J., \& Sanwal, D. 2000, ApJ, 531, L53

Pechenick, K. R., Ftaclas, C. Cohen, J. M. 1983, ApJ, 274, 846

Perna B Hernquist, L., \& Narayan R. 2000, ApJ, in press astro$\mathrm{ph} / 9912297$

Riffert, H, Nolert, H.-P., Kraus, U., \& Ruder, H. 1993, ApJ, 406, 185

Sugizaki, M., et al. 1997, PASJ, 49, L25

Thompson, C., \& Duncan, R. C. 1995, MNRAS, 275, 255

. 1996, ApJ, 473, 322

Torii, K., Kinugasa, K., Katayama, K., Tsunemi, H., \& Yamauchi, S. $1998,503,843$

van Paradijs, J., Taam, R., \& van den Heuvel, E. P. J. 1995, A\&A, 299, L41

Wiringa, R. B., Ficks, V., \& Fabrocini, A. 1988, Phys. Rev., C38, 1010

Zavlin, V.E. et al. 1995, A\&A 297, 441

Zavlin, V.E., Pavlov, G. G., \& Trumper, J. 1998, A\&A, 331, 212 